\title{
Prevalence of calcium sensing receptor autoantibodies in patients with sporadic idiopathic hypoparathyroidism
}

\author{
Ravinder Goswami, Edward M Brown ${ }^{1}$, Narayana Kochupillai, Nandita Gupta, Rajni Rani ${ }^{2}$, Olga Kifor ${ }^{1}$
} and Naibedya Chattopadhyay ${ }^{1}$

Department of Endocrinology and Metabolism, All India Institute of Medical Sciences, New Delhi 110029, India, ${ }^{1}$ Division of Endocrinology, Diabetes and Hypertension, and Membrane Biology Program, Department of Medicine, Brigham and Women's Hospital and Harvard Medical School, Boston, Massachusetts, USA and ${ }^{2}$ National Institute of Immunology, New Delhi, India

(Correspondence should be addressed to R Goswami; Email: gosravinder@hotmail.com)

\begin{abstract}
Objective: The pathogenesis of sporadic idiopathic hypoparathyroidism is unclear. The calcium sensing receptor (CaSR) plays a pivotal role in extracellular calcium homeostasis and is the candidate autoantigen in hypoparathyroidism associated with autoimmune polyglandular endocrinopathy syndrome. We therefore looked for antibodies $(\mathrm{Ab})$ against the CaSR in patients with sporadic idiopathic hypoparathyroidism and their association, if any, with the major histocompatibility complex (MHC) class II human leukocyte antigen (HLA)-DR haplotypes.

Methods: The subjects included 51 patients with sporadic idiopathic hypoparathyroidism and 45 healthy controls. Investigations included computerised tomography, serum calcium, phosphorus, thyroxine, TSH, cortisol, intact parathyroid hormone (iPTH), ACTH and thyroid peroxidase (TPO) and adrenal antibodies. The CaSRAb were assayed in patients' sera by Western blot. Genotyping of the HLA-DR locus was performed using PCR and sequence-specific oligonucleotide probes.

Results: Intracranial calcification and cataract were present in $76.5 \%$ and $41.1 \%$ of the patients respectively and $62.7 \%$ had convulsions. Autoantibodies against the $168 \mathrm{kDa}$ CaSR protein were demonstrated in the serum of $49.0 \%$ of the patients and in $13.3 \%$ of the controls $(P<0.001)$. Pre-incubating serum samples from the CaSRAb-positive patients with parathyroid membrane produced a $90 \%$ decrease in the band intensity. HLA-DRB1*01 and DRB1*09 alleles were significantly associated with idiopathic hypoparathyroidism (relative risk of $7.8, P=0.001$ ). The frequency of HLA-DRB $1 * 09$ and DRB1*10 alleles tended to be higher in patients positive for the CaSRAb. There was no significant difference in the frequency of occurrence of convulsions, cataract, intracranial calcification, calcium:phosphorus ratio, and iPTH levels between patients with and without CaSRAb. Conclusion: $49.0 \%$ of the patients studied had serological evidence of organ-specific autoimmunity against the CaSR protein. The occurrence of CaSRAb and the HLA-DR associations imply an autoimmune component to the disease, but the primary role of the CaSRAb in the pathogenesis of the disease needs to be assessed further.
\end{abstract}

European Journal of Endocrinology 150 9-18

\section{Introduction}

Idiopathic hypoparathyroidism is a heterogeneous group of disorders clinically characterised by the presence of tetany, cataract and basal ganglia calcification in association with hypocalcaemia, hyperphosphataemia and low or inappropriately normal serum intact parathyroid hormone (iPTH) $(1-5)$. The pathogenesis of sporadic idiopathic hypoparathyroidism has not yet been explained. In 1966, Blizzard et al. using indirect immunofluorescence, had reported the presence of parathyroid autoantibodies in patients with idiopathic hypoparathyroidism, but the organ-specific nature of these antibodies has been a subject of controversy
(6-9). Recently, the calcium sensing receptor (CaSR), which is a G-protein-coupled receptor, has been shown to play a pivotal role in the maintenance of the extracellular calcium homeostasis, which it does by sensing the circulating calcium levels and regulating the PTH synthesis and release $(10-13)$. Li et al. have reported the presence of autoantibodies against the CaSR among patients with hypoparathyroidism, in the majority of whom it was associated with the type 1 autoimmune polyglandular syndrome (APS-1) (14). However, to our knowledge there is no report that has investigated patients with sporadic idiopathic hypoparathyroidism for the presence of similar autoantibodies. Therefore, we studied the prevalence of CaSR 
autoantibodies (CaSRAb) in a group of patients with sporadic idiopathic hypoparathyroidism without associated APS-1 and examined their association with the major histocompatibility complex (MHC) class II human leukocyte antigen (HLA)-DR and with the clinical and biochemical indices of the disease.

\section{Subjects and methods}

\section{Subjects}

The subjects included 51 consecutive patients with hypoparathyroidism (29 males, 22 females) who attended the Endocrine Clinic at the All India Institute of Medical Sciences (AIIMS), New Delhi, from 1998 to 2002 . Their mean age at presentation was $33.1 \pm$ 12.8 years and the mean duration of their illness was $7.0 \pm 8.4$ years. The diagnosis of idiopathic hypoparathyroidism was based on the presence of hypocalcaemia and hyperphosphataemia in association with an inappropriately normal or subnormal level of serum iPTH. Patients with post-surgical hypoparathyroidism were excluded. None of the patients had a family history suggestive of hypoparathyroidism or adrenal insufficiency or clinical features of mucocutaneous candidiasis. The type 1 autoimmune polyglandular syndrome was excluded by demonstrating a normal adrenocorticotrophin (ACTH) value in all the 38 of 51 patients in whom fresh plasma samples could be obtained for ACTH assay. The $0800 \mathrm{~h}$ serum cortisol was also normal in all the subjects and there were no adrenal cortical autoantibodies observed using the indirect immunofluorescence technique described earlier (15). Six of the 51 patients $(11.7 \%$; 5 females and 1 male) had coexistent autoimmune disorders as evidenced by overt hypothyroidism in three, type 1 diabetes mellitus in one, alopecia areata with thyroid peroxidase antibody (TPOAb) positivity in one and TPOAb positivity alone in one. The study was approved by the Research and Ethics Committee of the AIIMS and written informed consent for participation was obtained from all the subjects. Clinical symptoms, including the duration of the illness and the presence of tetany, convulsions, and cataract were noted. Noncontrast computerised tomography of the brain was carried out for intracranial calcification. Details of one patient with reversible hypocalcaemic peripheral neuropathy have been published earlier (16). A basal blood sample was drawn after an overnight fast $(0800 \mathrm{~h})$ for the estimation of serum calcium and phosphorus and for the assay of iPTH (DiaSorin, Inc., Stillwater, MN, USA), total thyroxine $\left(\mathrm{T}_{4}\right)$, thyrotrophin (TSH) (Medicorp Inc., Montreal, Quebec, Canada), cortisol (Immunotech, Marseille, France) and TPOAb (Immunotech, Prague, Czech Republic), plasma ACTH (DiaSorin, Minnesota, USA), as well as for CaSRAb assays. Serum calcium and phosphorus levels were measured using the standard techniques used in our laboratory (17). All the samples were assayed for hormones in a single batch. The controls included 45 healthy subjects (26 males, 19 females, mean age $42 \pm 15$ years). The normal range for the basal hormone values were as follows: serum $\mathrm{T}_{4}=52-$ $167 \mathrm{nmol} / \mathrm{l}, \quad \mathrm{TSH}=0.3-4.0 \mathrm{mU} / \mathrm{l}, \quad$ cortisol $=260-$ $720 \mathrm{nmol} / \mathrm{l}, \quad$ iPTH $=13-54 \mathrm{ng} / \mathrm{l}, \quad$ ACTH $=1.3-12.4$ pmol/l. Intra-assay coefficients of variation ranged from $4 \%$ to $8 \%$ for the measurement of these hormones. As specified by the manufacturers of the TPOAb-RIA kit, a serum TPOAb titre $>100 \mathrm{IU} / \mathrm{ml}$ was considered positive.

\section{Western blot for the CaSRAb}

The presence of antibodies against the calcium sensing receptor was determined using Western blot analysis $(11,18)$. Briefly, parathyroid adenoma tissues were obtained at surgery from patients with primary hyperparathyroidism who underwent parathyroidectomy. The tissues were snap frozen in liquid $\mathrm{N}_{2}$ and were homogenised in a glass homogeniser with a teflon pestle in ice-cold lysis buffer in a 1:2 ratio by volume of sample to lysis buffer $(50 \mathrm{mmol} / \mathrm{l}$ Tris-HCl (pH 7.4); $0.25 \mathrm{~mol} / \mathrm{l}$ sucrose; $1 \mathrm{mmol} / \mathrm{l}$ EGTA, EDTA, PMFS and $10 \mu \mathrm{g} / \mathrm{ml}$ aprotonin, leupeptin and pepstatin (USB, Cleveland, OH, USA)). The nuclei and cell debris were pelleted by centrifugation at $800 \times \boldsymbol{g}$ and the supernatant then recentrifuged at $15000 \times \boldsymbol{g}$ to remove the mitochondrial fraction. The supernatant was finally sedimented by centrifugation at $45000 \times \boldsymbol{g}$ for $1 \mathrm{~h}$, and the pellet suspended in $2 \mathrm{ml} 1 \%$ Triton X-100 lysis buffer. All the centrifugations were carried out at $4^{\circ} \mathrm{C}$. The protein content of the solubilised membrane fraction was estimated using Bradford's reagent and the readings taken at $595 \mathrm{~nm}$ (Shimadzu, UV160). Parathyroid adenomas have been reported to show a variable, often consistent reduction in the CaSR expression (19). Therefore, in this study we used only those parathyroid adenoma tissues whose cell membranes showed a relatively high level of expression of the CaSR for screening the patients' sera for the CaSRAb. This level of expression of the CaSR in the different adenoma membranes was assessed by first staining all our membrane preparations using a commercially available anti-calcium sensing receptor antibody on Western blot (PA1-934, Affinity BioReagents, Inc., Golden, CO, USA). Only those membrane preparations which displayed a CaSR protein band of good intensity on Western blot analysis were subsequently utilised. The membrane fractions thus prepared were made into aliquots of $100 \mu \mathrm{l}$ each (containing $2 \mu \mathrm{g} / \mu \mathrm{l}$ protein) and were stored at $-20^{\circ} \mathrm{C}$ until used. For assay, one aliquot of the fraction prepared as described above was mixed in a ratio of 1:5 with $5 \times$ Laemmli buffer (0.32 Tris pH 6.8), 5\% (w/v) SDS, 25\% (v/v) glycerol, $0.1 \%(\mathrm{w} / \mathrm{v})$ bromophenol blue, $143 \mathrm{mmol} / \mathrm{l}$ $\beta$-mercaptoethanol (Sigma, St Louis, MO, USA), and 
$20 \mu \mathrm{g}$ of the protein were loaded in each well for electrophoresis without prior boiling. The proteins were fractionated using SDS-PAGE mini gels $(8 \times 7 \mathrm{~cm}$ with $5 \%$ resolving and $4 \%$ stacking gels; Hoeffer, San Francisco, CA, USA), transferred to PVDF membranes and probed overnight with patients' sera (1:50 dilution with $1 \%$ Blotto/PBS) at $4{ }^{\circ} \mathrm{C}$ using a deca-probe incubation manifold as described earlier (18). Anti-human (1:1000 dilution) and anti-rabbit IgG conjugated with horseradish peroxidase (1:500 dilution, DAKO, Glostrup, Denmark) were used as second antibodies (incubation time, $1.5 \mathrm{~h}$ ) and 3-amino-9-ethylcarbizole in $0.1 \mathrm{~mol} / \mathrm{l}$ acetate buffer ( $\mathrm{pH} 5.2$ ) was used for optimal colour development (AEC, DAKO, Carpinteria, CA, USA) . In addition to the patients' sera, each blot also included 4 lanes, 2 of these containing various positive controls, pre-stained protein molecular weight markers, and a negative control containing only $1 \%$ Blotto/PBS. The various positive controls used included (i) a commercially procured antibody against the CaSR (PA1-934), (ii) a polyclonal, affinity-purified, antiCaSR antibody (code: 4637) raised in rabbits as described earlier (19); both the above antibodies recognised the $168 \mathrm{kDa}$ as well as the $151 \mathrm{kDa}$ isoforms of the CaSR, and (iii) serum from a patient with hypoparathyroidism which contained an antibody strongly positive for only the $168 \mathrm{kDa}$ CaSR protein. In order to minimise the introduction of bias in the interpretation of results, the blots were read independently by two investigators, one of whom (EMB) was kept blind to the identity of the samples (see Fig. 1).

Specificity of the CaSR antibody in the patients' sera was confirmed by doing neutralisation experiments demonstrating the blocking of these antibodies using antigens obtained from two different sources. (i) A

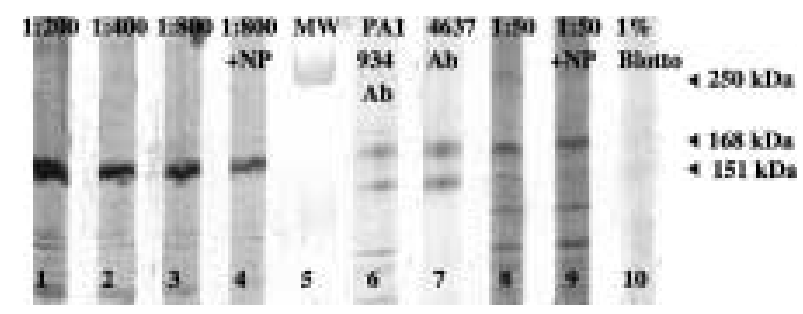

Figure $1 \mathrm{Immunoblotting} \mathrm{of} \mathrm{proteins} \mathrm{from} \mathrm{membranes} \mathrm{prepared}$ from human parathyroid adenomas. A $5 \%$ SDS-PAGE Western blot using parathyroid membrane protein ( $20 \mu \mathrm{g}$ protein/lane) shows seropositivity against 168 and $151 \mathrm{kDa}$ CaSR proteins by CaSR-antibody-positive control serum (PA1-934) in lane 6 and another positive control serum (4637 antibodies) in lane 7; negative control ( $1 \%$ blotto/PBS, blank) with no seropositivity against the 168 and $151 \mathrm{kDa}$ proteins is shown in lane 10. A patient with hypoparathyroidism showing CaSRAb against $168 \mathrm{kDa}$ in 1:200, $1: 400$ and $1: 800$ dilution (lanes 1,2 and 3 respectively) and $28.4 \%$ neutralisation of seropositivity at a 1:800 dilution with neutralizing peptide (NP) PEP-009 (lane 4), and a second patient with hypoparathyroidism showing seropositivity in 1:50 dilution against 168 and $151 \mathrm{kDa}$ proteins (lane 8 ) and $11.1 \%$ reduction of

$151 \mathrm{kDa}$ band positivity with NP-PEP-009 (lane 9) are also shown. Lane 5, molecular weight (MW) markers. commercially available neutralizing peptide PEP-009, against which the PA1-934 antibody had been developed (Affinity BioReagents, Inc.); PEP-009 is a 16 amino acid synthetic peptide whose sequence is derived from and is identical to the amino terminal end of the rat CaSR protein (12-27 amino acids: A-L-A-W-H-S-S-A-Y-G-P-D-Q-R-A-Q). PEP-009 binds and blocks the rat as well as the human CaSRAb (20). (ii) A human parathyroid adenoma membrane preparation which showed a good expression of the CaSR on Western blot analysis as described above. Serum samples obtained from two patients with hypoparathyroidism, which were most strongly positive for autoantibodies against the 168 and $151 \mathrm{kDa}$ CaSR proteins were first serially diluted to determine the lowest titre at which they tested positive for the CaSRAb. The neutralisation of these antibodies was then carried out by incubating the sera at this titre with $30 \mu \mathrm{g}$ of the peptide PEP-009. The difference in the integrated density value of the CaSRAb band obtained in the presence and in the absence of the PEP-009 peptide was measured by densitometry using the image analyser software, Alphaease (Chemilmager 4400, Alpha Innotech Corp., San Leandro, CA, USA). In order to demonstrate specific neutralisation of the CaSRAb by the parathyroid adenoma membrane preparation, $10 \mu \mathrm{l}$ serum from a patient whose serum was strongly positive for CaSRAb were first incubated overnight with an excess of the membrane protein on a rocker at $4^{\circ} \mathrm{C}$, before carrying out the Western blot analysis $(200 \mu \mathrm{g}$ membrane protein in $500 \mu \mathrm{l}$ lysis buffer). The neutralisation was assessed by densitometry as described above. Similar neutralisation experiments to assess the specificity of the CaSRAb were also performed using membrane preparations (200 $\mu \mathrm{g}$ each) from human liver and skeletal muscle tissues obtained at autopsy (see Fig. 2B).

\section{Western blot for CaSRAb using rat liver and myocardial tissue, as well as human liver and skeletal muscle membrane preparations}

Since there are some reports in the literature showing weak expression of the CaSR in the rat hepatocyte and myocardial tissues $(21,22)$, we also examined these tissues along with human liver and skeletal muscle membranes, for expression of the calcium sensing receptor and to investigate their ability to detect the CaSRAb in the patients' sera (see Fig. 3A and B). The procedure adopted for extraction of membrane preparations from the above-mentioned tissues was essentially the same as described above for the parathyroid tissue membranes.

\section{Genotyping for MHC class II, HLA-DR locus}

In 36 of the 51 patients with hypoparathyroidism, DNA was extracted from $10 \mathrm{ml}$ blood using the standard 
(A)

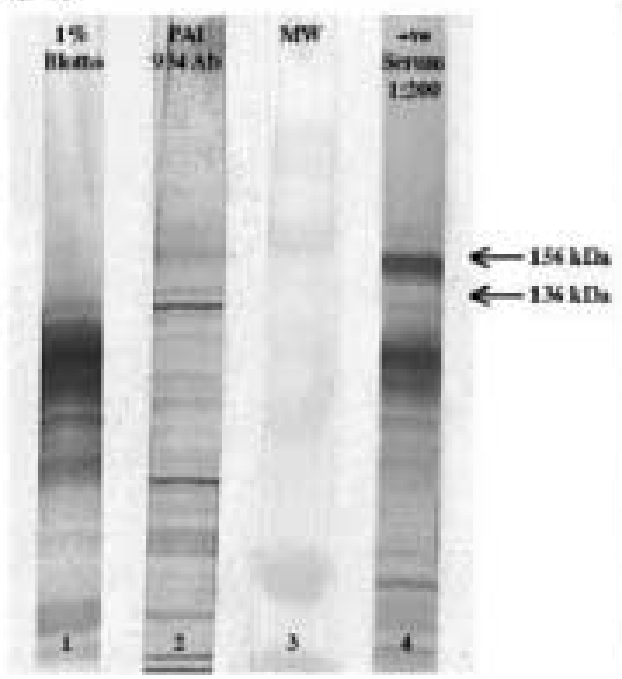

(B)

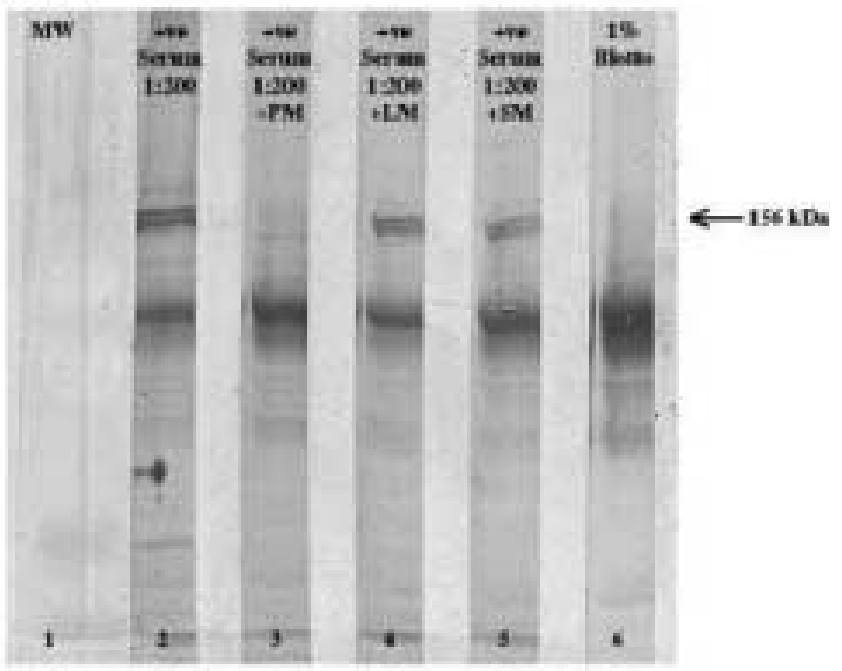

Figure 2 (A) Heterogeneity of the molecular weight of CaSR antigens. A $5 \%$ Western blot using parathyroid membrane protein $(20 \mu \mathrm{g}$ protein/lane) shows CaSRAb against $156 \mathrm{kDa}$ antigen in a patient with idiopathic hypoparathyroidism in a serum dilution of $1: 200$ in lane 4, and PA1-934 shows positivity against 156 and $136 \mathrm{kDa}$ antigens in lane 2. Lane 1, blank and lane 3, molecular weight (MW) markers. The membrane preparation used in this experiment belonged to a different patient undergoing parathyroid adenectomy than that shown in Fig. 1. However, the sera in lane 4 of Fig. 2A, lane 2 of Fig. $2 B$ and in lanes $1-3$ of Fig. 1 were from the same patient. (B) Specific neutralisation of the CaSRAb in a patient with idiopathic hypoparathyroidism with parathyroid membrane protein. A $5 \%$ SDS-PAGE Western blot using various membrane protein preparations ( $20 \mu \mathrm{g}$ protein/lane) shows seropositivity against $156 \mathrm{kDa}$ in a patient with hypoparathyroidism (1:200 dilution, lane 2) and 90\% neutralisation of CaSRAb band positivity after overnight preincubation of the serum with parathyroid membrane (lane 3 ); only $16 \%$ and $28 \%$ neutralisation of CaSRAb positivity following preincubation of the CaSRAb-positive sera with cell membrane preparations from human liver (LM, lane 4) and human skeletal muscle (SM, lane 5) is seen. Lanes 1 and 6 are MW markers and blank (1\% Blotto/PBS) respectively.

phenol-chloroform extraction technique (23). The HLA-DR genotyping was carried out using PCR (Applied Biosystem) followed by hybridisation with sequence-specific oligonucleotide probes (PCR-SSOP). Briefly, amplification of the second exon of the HLADRB1 gene was performed using the primers described earlier (24). The PCR products were run on $1 \%$ agarose gels to confirm the amplification and were then dot blotted on Hybond $\mathrm{N}(+)$ membranes (Amersham, Life Sciences Inc., Chicago IL, USA) and UV cross linked. The membranes with the dot blots were then hybridised with ${ }^{32}$ P-labelled probes for DRB $(1-18)$ generic. Differences in the frequency of the presence of specific HLADR alleles in patients with hypoparathyroidism as a group and when stratified according to CaSRAb positivity were compared with the data base already available to us from 94 healthy controls drawn from the same North Indian ethnic background (24).

\section{Statistical analysis}

Chi-square analysis was used to compare the frequency of occurrence of CaSRAb positivity between the patient and the control groups. The Chi-square test and the Student's t-test were used to compare the frequency of occurrence and the mean of the different variables respectively, between the group of patients with and without CaSRAb. A $P$ value of $<0.05$ was considered statistically significant. For HLA analysis, the $P$ values were calculated using Fisher's exact test, and the results obtained were corrected by multiplying by the number of DR alleles tested. Relative risks and odds ratios were calculated using Woolf's method with Haldanes's modification (25).

\section{Results}

A history of convulsions, cataract and intracranial calcification on imaging was present in 32 (62.7\%), 21 $(41.1 \%)$ and $39(76.5 \%)$ of the 51 patients respectively. Subjects with intracranial calcification had a higher frequency of occurrence of cataract when compared with those without calcification $(19 / 39,48.7 \%$ vs $2 / 12,17.7 \%, P=0.048)$. The mean duration of the illness was longer in patients who had intracranial calcification or cataract as compared with patients without these complications $(9.0 \pm 9.5$ years vs $2.4 \pm 4.1$ years, $P=0.002$ and $11.6 \pm 10.4$ years vs $4.4 \pm 6.4$ years, $P=0.01$ respectively). On linear regression analysis of the data in models where age of onset of symptoms, duration of illness and serum calcium levels were considered as independent variables, only the duration of the illness explained the presence of the variations observed in the frequency of occurrence of cataract or basal ganglia calcification (dependent variables) 

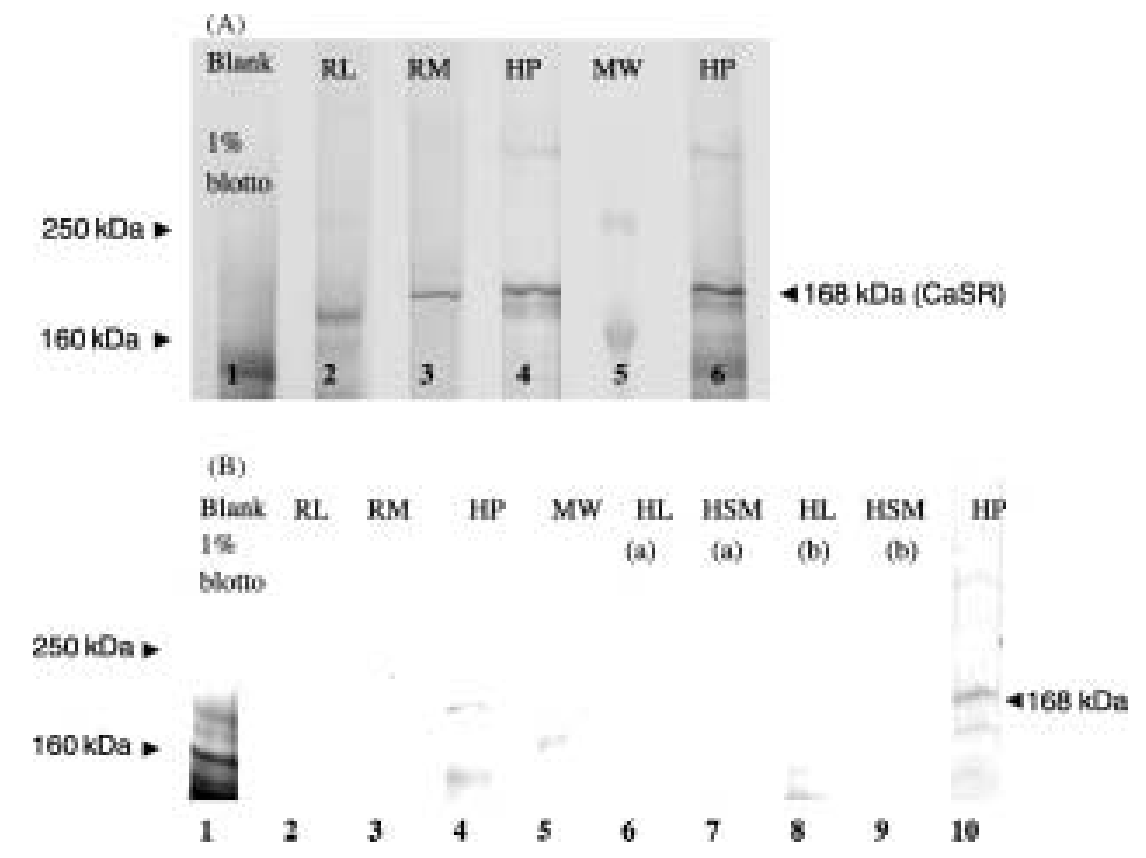

Figure 3 (A) Immunoblot of membrane proteins (20 $\mu \mathrm{g}$ protein/lane) prepared from rat liver (RL, lane 2), rat myocardial tissue (RM, lane 3) and human parathyroid adenoma (HP, lanes 4 and 6). The 5\% SDS-PAGE Western blot probed with the commercial CaSRAbpositive control serum (PA1-934) shows the presence of the $168 \mathrm{kDa}$ CaSR protein in lanes $3(\mathrm{RM})$ and 4 (HP) and is also positive for a $162 \mathrm{kDa}$ antigen in lane $2(\mathrm{RL})$. CaSRAb-positive serum (1:50 dilution) from a patient with hypoparathyroidism also shows reactivity against the $168 \mathrm{kDa}$ CaSR protein in lane 6 (HP). Lanes 1 and 5 are blank (1\% Blotto/PBS) and molecular weight (MW) markers respectively. (B) Immunoblot of membrane proteins $(20 \mu \mathrm{g}$ protein/lane) prepared from rat liver (RL, lane 2), rat myocardial tissue (RM, lane 3), human parathyroid adenoma (HP, lanes 4 and 10), human liver and skeletal muscle tissue obtained from two different autopsies (HL a and b, lanes 6 and 8; HSM a and b, lanes 7 and 9). A 5\% SDS-PAGE Western blot shows seropositivity against $168 \mathrm{kDa}$ by PA1-934 CaSR antibody in lane 10. CaSRAb-positive serum (1:50 dilution) from a patient with hypoparathyroidism also shows positivity against the $168 \mathrm{kDa}$ CaSR protein in lanes 4 (HP), 7 and 9 (HSM a and b respectively, weekly positive). The same patient's serum showed no seropositivity against CaSR protein in lanes 2 (RL), 3 (RM) and in lanes 6 and 8 (HL a and b respectively). Lanes 1 and 5 are blank (1\% Blotto/PBS) and MW markers respectively.

respectively $(15.4 \pm 45.7 \%, P<0.01$ and $16.7 \pm 40.2$, $P<0.002)$.

Western blot analysis demonstrated the presence of antibody against the CaSR in 25 of the 51 (49.0\%) patients with hypoparathyroidism. The frequency of occurrence of the CaSRAb among the 45 subjects without any associated autoimmune endocrinopathy $(51.1 \%)$ was similar to that observed for the whole group of 51 patients $(49.0 \%)$. Twenty-one of the 25 patients showing CaSRAb were seropositive only for the $168 \mathrm{kDa}$ protein while four were positive for both the 168 as well as the $151 \mathrm{kDa}$ protein (Table 1 , Fig. 1). In contrast, only $6 / 45$ (13.3\%) of the controls studied had autoantibodies against the $168 \mathrm{kDa}$ CaSR (patients vs controls $P<0.001$ ). Following incubation with an excess of the synthetic neutralising peptide, PEP-009, whose sequence is identical to the amino terminal end of the rat CaSR protein and which also binds and blocks the human CaSRAb (20), there was a $28.4 \%$ and $11.1 \%$ reduction in the integrated density value (IDV) of the $168 \mathrm{kDa}$ and $151 \mathrm{kDa}$ bands respectively (Fig. 1, lane 3 vs lane 4 and lane 8 vs lane 9).

Table 1 Calcium sensing receptor and thyroid peroxidase autoantibodies status and HLA-DR associations in patients with idiopathic hypoparathyroidism and in healthy controls.

\begin{tabular}{|c|c|c|c|}
\hline Parameters & Patients & Healthy controls & $P$ value \\
\hline Overall CaSRAb positivity & $25 / 51(49.0 \%)$ & $6 / 45(13.3 \%)$ & $<0.001$ \\
\hline CaSRAb $168 \mathrm{kDa}$ alone & $21 / 51(41.1 \%)$ & $5 / 45(11.1 \%)$ & 0.0021 \\
\hline CaSRAb both $168 \& 151 \mathrm{kDa}$ & $4 / 51$ & Nil & 0.058 \\
\hline CaSRAb $151 \mathrm{kDa}$ alone & Nil & Nil & - \\
\hline Thyroid peroxidase antibody positivity & $3(5.8 \%)$ & 0 & 0.24 \\
\hline Frequency of HLA-DRB1*01 allele & $4 / 36(11.1 \%)$ & $1 / 94(1.1 \%)$ & 0.020 \\
\hline Frequency of HLA-DRB1*09 allele & $4 / 36(11.1 \%)$ & $2 / 94(2.1 \%)$ & 0.049 \\
\hline Frequency of HLA-DRB1*01+DRB1*09 alleles & $8 / 36(22.2 \%)$ & $3 / 94(3.2 \%)$ & 0.0015 \\
\hline
\end{tabular}


Figure 2A and $\mathrm{B}$ displays the results of experiments showing specific neutralisation of the CaSRAb in a patient with idiopathic hypoparathyroidism. Following preincubation of the serum with an excess of parathyroid membrane proteins, the IDV of the CaSRAb band was reduced by $90 \%$ on blot densitometry. In contrast, the IDV of the CaSRAb band was reduced by only $16 \%$ and $28 \%$ respectively when liver and skeletal muscle membrane preparations were used. This reduction in IDV could be explained by the relatively weak expression of the CaSR in the human liver and skeletal muscle preparations (Fig. 3A and B). In the parathyroid adenoma membrane preparation used for neutralisation experiments, both the commercial anti-calcium sensing receptor antibody PA1-934, as well as the $\mathrm{CaSRAb}$ from the patient with hypoparathyroidism, reacted with the CaSR antigen protein at molecular weights corresponding to $156 \mathrm{kDa}$ and $136 \mathrm{kDa}$ and not with $168 \mathrm{kDa}$ and $151 \mathrm{kDa}$, as observed in earlier preparations (Fig. 2A and B).

Both the rat liver and the rat myocardial membrane preparations showed CaSR expression as demonstrated by their reactivity with the PA1-934 CaSR specific antibodies (Fig. 3A). However, serum obtained from a patient with hypoparathyroidism, which had reacted strongly with the $168 \mathrm{kDa}$ CaSR protein in a human parathyroid membrane preparation, did not react against the CaSR protein present in rat liver and myocardial membranes, possibly indicating the speciesspecific nature of the CaSRAb (Fig. 3B).

There was no significant difference in the frequency of occurrence of the TPOAb positivity between the patient and the control groups (Table 1). Table 2 gives the details of the various other indices related to hypoparathyroidism, which were also examined in patients with and without CaSRAb. Among the hypoparathyroid patients, there were no significant differences observed between the group of CaSRAb-positive and
CaSRAb-negative patients with regard to the male to female ratio, frequency of occurrence of tetany, convulsions, cataract, basal ganglia calcification, mean serum calcium, serum phosphorus or their ratios, serum iPTH values or the frequency of presence of TPOAb. The six subjects belonging to the control group who also demonstrated serological evidence of CaSRAb were asymptomatic and had no clinical or biochemical evidence of hypoparathyroidism.

Of the 18 HLA-DRB alleles analysed, the frequency of occurrence of HLA-DRB $1 * 01$ or DRB $1 * 09(n=4$ each $)$ was significantly higher among the 36 patients with hypoparathyroidism when compared with the frequency of occurrence of the same alleles $(n=1$ and 2 respectively) in the 94 healthy controls studied who were derived from the same ethnic background $(P=0.001, P$ corrected for 18 alleles tested $=0.02)$ with Haldanes' corrected relative risk of 7.8. The frequency of occurrence of the HLA-DRB $1 * 09$ or DRB1 $* 10$ allele $(n=4$ each) was higher among patients with hypoparathyroidism who were positive for the CaSRAb as compared with those who were negative for the antibody $(n=1, P=0.02$, corrected $P=0.36$, odds ratio $=5.2$ ) (Table 1$)$. However, there was no significant difference in the frequency of occurrence of any of the specific alleles of the generic HLADR when the 36 patients with hypoparathyroidism were segregated on the basis of the presence or absence of convulsions, cataract or intracranial calcification.

\section{Discussion}

In the present study, the incidence of intracranial calcification, convulsions, and cataract as well as the male to female ratio in the cohort of 51 patients with idiopathic hypoparathyroidism, were comparable to what has been reported earlier in the literature (26-29).

Table 2 Study variables in relation to calcium sensing receptor autoantibody status in patients with idiopathic hypoparathyroidism.

\begin{tabular}{|c|c|c|c|}
\hline Parameters & CaSRAb + ve $(n=25)$ & CaSRAb - ve $(n=26)$ & $P$ value \\
\hline Sex (Male:Female) & $15: 10$ & $14: 12$ & 0.65 \\
\hline Age (years) & $31.9 \pm 12.4$ & $34.3 \pm 13.5$ & 0.50 \\
\hline Duration of symptoms (years) & $8.3 \pm 9.3$ & $6.2 \pm 7.7$ & 0.38 \\
\hline BMI $\left(\mathrm{kg} / \mathrm{m}^{2}\right)$ & $20.7 \pm 4.1$ & $22.0 \pm 5.1$ & 0.68 \\
\hline History of convulsions & $19 / 24(76.0 \%)$ & $13 / 24(50.0 \%)$ & 0.054 \\
\hline History of tetany & $19(76.0 \%)$ & $17(65.4 \%)$ & 0.40 \\
\hline Cataract & $12(48.0 \%)$ & $9(34.6 \%)$ & 0.33 \\
\hline Serum calcium (mmol/l) & $1.44 \pm 0.27$ & $1.44 \pm 0.20$ & 0.97 \\
\hline Serum inorganic phosphorus (mmol/l) & $2.03 \pm 0.45$ & $2.1 \pm 0.32$ & 0.40 \\
\hline Intracranial calcification & $20(80 \%)$ & $19(73.0 \%)$ & 0.61 \\
\hline Serum intact PTH (ng/l) & $10.0 \pm 9.2$ & $9.7 \pm 7.0$ & 0.88 \\
\hline Thyroid peroxidase antibody positivity & $2(8.0 \%)$ & Nil & 0.99 \\
\hline Hypothyroidism & Nil & $3(11.5 \%)$ & 0.23 \\
\hline Frequency of HLA-DRB1*09 allele & $4 / 19(21.0 \%)$ & $0 / 17$ & 0.065 \\
\hline Frequency of HLA-DRB1*10 allele & $4 / 19(21.0 \%)$ & $1 / 17(5.8 \%)$ & 0.20 \\
\hline Frequency of HLA-DRB1*09+DRB1*10 alleles & $8 / 19(42.1 \%)$ & $1 / 17(5.8 \%)$ & 0.014 \\
\hline
\end{tabular}

BMI, body mass index. 
The present study also demonstrated the coexistence of intracranial calcification and cataract, both of which were individually associated with a longer duration of the illness. However, the duration of the illness could explain the variation in the occurrence of intracranial calcification and cataract in only $15-16 \%$ of the patients. These findings suggest a role for other factors in their aetiology.

In 1966, Blizzard et al. using indirect immunofluorescence had reported the presence of parathyroid autoantibodies in 33\% of patients with idiopathic hypoparathyroidism (6). However, Swana et al. (1977) and Betterle et al. (1985) using similar techniques demonstrated anti-mitochondrial antibodies staining the mitochondria-rich oxyphil cells of the parathyroid gland and suggested that these could be the cause of the seropositivity observed in patients with idiopathic hypoparathyroidism, which had been reported earlier $(8,9)$. Using Western blot assays, Fattorossi et al. (1988) first described the presence of the $200 \mathrm{kDa}$ and $130 \mathrm{kDa}$ autoantigens in the parathyroid endothelial/epithelial cells of six patients with idiopathic hypoparathyroidism (30). Subsequently, Li et al. in 1996 identified the CaSR as an autoantigen and demonstrated antibodies against this receptor in $56 \%$ of 25 patients with hypoparathyroidism, 8 of whom were adults with hypoparathyroidism and coexisting thyroiditis and 17 were children with type 1 autoimmune polyglandular syndrome (14).

The inclusion of hypoparathyroidism among the autoimmune endocrinopathies associated with the autoimmune polyglandular syndrome, and the demonstration of CaSR autoantibodies in these patients indirectly suggests that similar autoimmune mechanisms might be operative in subjects with idiopathic hypoparathyroidism. Patients who have endocrinopathies linked with the autoimmune polyglandular syndrome often demonstrate a higher prevalence of organ-specific autoantibodies and exhibit autoantigens of a different type as compared with patients having the same endocrinopathies occurring in isolation without the polyglandular syndrome $(31,32)$. For example, Uibo et al. (31) reported the presence of autoantibodies against the cytochrome P450c17, P450scc and/or P450c21 hydroxylases in $81 \%$ of their patients with Addison's disease associated with the autoimmune polyglandular syndrome while, in contrast, only $25 \%$ of the patients with isolated Addison's disease demonstrated these autoantibodies, which were found to be directed exclusively against P450c21 hydroxylase $(31,32)$. In the present study, the majority of patients had sporadic idiopathic hypoparathyroidism without coexisting autoimmune endocrinopathy (45/51) and $51.1 \%$ of them had serological evidence of autoantibodies against the CaSR.

In the present study, six of the 45 healthy controls (13.3\%) also showed CaSR autoantibodies. Blizzard et al. (1966) had also reported $6 \%$ of their controls to be positive for parathyroid autoantibodies (6). On the other hand, Li et al. did not detect CaSR autoantibodies in any of the 22 healthy controls studied by them (14). These variations observed in the prevalence of parathyroid autoantibodies among healthy controls may be due to differences in the number of controls studied. Interestingly, in the present study, none of the six controls who were positive for CaSR autoantibodies showed clinical or biochemical signs of hypoparathyroidism.

The CaSR protein can migrate to several different positions on SDS PAGE depending upon variations in the degree of its glycosylation or dimer formation (10, 12). Under non-reducing conditions, the $151 \mathrm{kDa}$ band represents the immature, high mannose, less glycosylated form of the CaSR protein, and the band corresponding to the $168 \mathrm{kDa}$ protein represents the monomeric, mature receptor which is fully glycosylated. The N-linked glycosylation of the CaSR protein is important for the normal expression of the receptor on the cell surface $(11-13)$. The protein band analogous to a molecular weight of $>250 \mathrm{kDa}$ represents the dimer of the CaSR (12). Thus, in the present study, all the 25 patients had autoantibodies demonstrated against the $168 \mathrm{kDa}$ CaSR autoantigen and only 4 of them had autoantibodies against the $151 \mathrm{kDa}$ protein. However, while conducting the neutralisation experiment for the CaSRAb when we used parathyroid adenoma membrane preparations obtained from different patients, the commercial calcium sensing receptor antibody PA1-934 as well as the CaSRAb from the strongly positive patient's sera reacted with proteins corresponding to a CaSR molecular weight of $156 \mathrm{kDa}$ and $136 \mathrm{kDa}$ instead of $168 \mathrm{kDa}$ and $151 \mathrm{kDa}$ as observed earlier (Fig. 2B). This reactivity of the CaSR autoantibody was reduced by $90 \%$ on incubation of the serum with an excess of parathyroid membrane protein, confirming that these autoantibodies were CaSR-protein specific (Fig. 2A). However, incubation of the serum samples with the 16 amino acid synthetic analogue of the extracellular part of the CaSR (PEP009) resulted in only a $28.4 \%$ decline in reactivity of CaSR antibody. The difference in the neutralisation of the CaSRAb observed when using parathyroid membrane preparation and PEP-009 peptide (extracellular part of the CaSR) suggests that these patients with idiopathic hypoparathyroidism may have a heterogeneous pool of autoantibodies with binding sites directed against different epitopes of CaSR (Fig. 1). Further, the CaSR autoantibody present in the serum of one of the patients with hypoparathyroidism appeared to be species specific, because although it had reacted strongly with the CaSR antigen in the human parathyroid membrane preparations, the antibody failed to react with the CaSR protein when membranes from rat liver or myocardial tissues were used (Fig. 3B).

In the present group of hypoparathyroid patients studied, we can only speculate whether the CaSR autoantibodies observed bring about the primary destruction 
of the parathyroid gland or whether these autoantibodies mark the end stage of a disease process which originally begins with damage to the parathyroid gland mediated by the Th1 lymphocytes with consequent activation of the local Th2 cells, leading to the gradual development of autoantibodies, a pattern of progression which may take place in predisposed individuals with specific class II haplotypes. Brandi et al. have demonstrated antibody-dependent cytotoxicity in cultured bovine parathyroid cells when exposed to serum from patients with autoimmune hypoparathyroidism and type 1 APS (3). Besides, Wortsman et al. have demonstrated $\mathrm{T}$ lymphocyte activation with increased natural killer cell activity but an absence of B-cell activation in patients with adult onset idiopathic hypoparathyroidism (2). Weak immunohistochemical reactivity for both MHC class I and class II antigens has been demonstrated in dispersed normal human parathyroid cells (33). While we could demonstrate HLA-DR associations in the whole cluster of patients with idiopathic hypoparathyroidism, the CaSRAb detected in the sera of these patients did not correlate with either the clinical manifestations or the biochemical abnormalities of the disease. In diseases where autoantibodies have a primary role in pathogenesis, the clinical and biochemical severity of the illness correlates with the degree of seropositivity, for example in Graves' disease and myasthenia gravis $(34,35)$. Thus, among the patients in the present study the absence of any correlation of the CaSRAb with the clinical or biochemical manifestations of the disease may either represent an epiphenomenon due to immune activation or might involve antibody-dependent cell-mediated cytotoxicity.

Alternatively, the CaSRAb might lead to a decline in the PTH release essentially by acting as an agonist for binding to the CaSR that is naturally expressed on the parathyroid cell membranes. However, such a phenomenon by itself would not bring about the parathyroid gland atrophy and lymphocytic infiltration that have been observed and reported at autopsy in a few patients with idiopathic hypoparathyroidism (4). While the above facts argue against a primary role and any functional significance of the CaSRAb among patients with idiopathic hypoparathyroidism, there is a recent report of CaSR blocking autoantibodies described in patients with a syndrome resembling familial hypocalciuric hypercalcemia (36). However, in order to reveal the pathogenetic mechanism(s) at work, further studies need to be done in vitro to examine the possible role of the CaSR autoantibody in altering the normal functioning of the G-protein-mediated intracellular signalling pathways used by this receptor, as well as an analysis of the Th1 and Th2 cytokine profiles among patients with idiopathic hypoparathyroidism. In the present study, the hypoparathyroidism observed in $51 \%$ of patients in whom serological evidence of CaSRAb could not be demonstrated would still be referred to as 'idiopathic hypoparathyroidism'. In this group, the existence of autoimmunity to antigens other than the CaSR or disappearance of the CaSRAb positivity are possible alternatives. Moreover, in the present cohort of patients, we also cannot rule out the theoretical possibility of the existence of activating mutations of the CaSR gene or altered cleavage of the PTH signal peptide due to a mutation in the preproPTH gene - both known causes of hypoparathyroidism. However, genetic mutations involving the CaSR and prepro-PTH gene have been reported among patients with familial isolated hypoparathyroidism and not in subjects with the sporadic form of hypoparathyroidism of the type reported in the present study $(37,38)$.

Multiple target gland involvement is frequent among patients with autoimmune endocrinopathies. In India, among patients with type 1 diabetes mellitus with no clinical and biochemical evidence of hypothyroidism, the prevalence of concurrent thyroid autoimmunity is $26 \%$ (39). In the present study, while we could document serological evidence of CaSRAb in half of the patients with hypoparathyroidism, the observed frequency of coexisting thyroid autoimmunity was only $9.8 \%$ which was not significantly different from that observed among the controls. This would suggest that patients with idiopathic hypoparathyroidism develop autoimmunity to the thyroid gland only infrequently.

The autoimmune nature of hypoparathyroidism warrants a study of its HLA associations. However, there is no data on HLA-DR associations among patients with idiopathic isolated hypoparathyroidism. In the present study the occurrence of the HLADRB1*01 or DRB1*09 alleles was significantly higher among the hypoparathyroid patients, with a relative risk of 7.8. Allelic variations were also demonstrated in relation to their CaSRAb status, with the frequency of HLA-DRB1*09 or DRB1*10 being higher in the CaSRAb-positive patients with an odds ratio of 5.2. However, the latter did not attain statistical significance. No similar association in allelic variation was observed when the patients were stratified on the basis of presence or absence of complications such as intracranial calcification or cataract. Further detailed studies involving associations with HLA class 1 and class II-DQB1, DQA1 and DPB1 alleles in the present group of patients might reveal such associations.

To conclude, the present study documents serological evidence of autoimmunity against a $168 \mathrm{kDa}$ CaSR protein in almost half (49\%) of a group of patients with sporadic idiopathic hypoparathyroidism who were investigated. The antibodies were demonstrable even up to six years after the onset of the disease. While CaSRAb seropositivity and HLA-DR associations support the autoimmune nature of the disease, the primary role of the CaSRAb in the pathogenesis of the idiopathic hypoparathyroidism needs to be further examined in view of the fact that the CaSRAb positivity did not segregate patients with regard to the frequency with which they developed convulsions, cataract, intracranial 
ganglia calcification, abnormal phosphorus/calcium ratio or any other clinical manifestations of the disease studied.

\section{Acknowledgements}

This work was supported by the Research Grant from the All India Institute of Medical Sciences (to R G) and grants from the National Institutes of Health (DK41415, DK52005 and DK438330) (to E M B), NPS Pharmaceuticals (to E M B), and the St Giles Foundation (to E M B). The authors acknowledge Dr S Chumber, Additional Professor, Department of Surgery, for providing parathyroid tissue and Dr Rajveer Singh, Scientist Department of Bio-statistics, AIIMS, Delhi for statistical analysis of the data.

\section{References}

1 Downs RW. The hypoparathyroid states. In The Parathyroid: Basics and Clinical Aspect, edn 2, pp 755-762. Eds JP Bilezikian, R Marcus \& MA Levine. Boston: Academic Press, 2001.

2 Wortsman J, McConnachie P, Baker JR Jr \& Mallette LE. T-lymphocyte activation in adult-onset idiopathic hypoparathyroidism. American Journal of Medicine $199292352-356$.

3 Brandi ML, Aurbach GD, Fattorossi A, Quarto R, Marx SJ \& Fitzpatrick LA. Antibodies cytotoxic to bovine parathyroid cells in autoimmune hypoparathyroidism. PNAS $1986 \quad \mathbf{8 3}$ $8366-8369$.

4 Boyce BF, Doherty VR \& Mortimer G. Hyperplastic parathyroiditis - a new autoimmune disease? Journal of Clinical Pathology 1982 $35812-814$.

5 Posillico JT, Wortsman J, Srikanta S, Eisenbarth GS, Mallette LE \& Brown EM. Parathyroid cell surface autoantibodies that inhibit parathyroid hormone secretion from dispersed human parathyroid cells. Journal of Bone and Mineral Research $1986 \mathbf{1}$ 475-483.

6 Blizzard RM, Chee D \& Davis W. The incidence of parathyroid and other antibodies in the sera of patients with idiopathic hypoparathyroidism. Clinical and Experimental Immunology $19661119-128$.

7 Irvine WJ \& Scarth L. Antibody to the oxyphil cells of the human parathyroid in idiopathic hypoparathyroidism. Clinical and Experimental Immunology $1969 \mathbf{4}$ 505-510.

8 Swana GT, Swana MR, Bottazzo GF \& Doniach D. A human-specific mitochondrial antibody - its importance in the identification of organ-specific reactions. Clinical and Experimental Immunology $197728517-525$.

9 Betterle C, Caretto A, Zeviani M, Pedini B \& Salviati C. Demonstration and characterization of anti-human mitochondria autoantibodies in idiopathic hypoparathyroidism and in other conditions. Clinical and Experimental Immunology $1985 \mathbf{6 2}$ 353-360.

10 Brown EM, Gamba G, Riccardi D, Lombardi M, Butters R, Kifor O et al. Cloning and characterization of an extracellular $\mathrm{Ca}^{(2+)}$-sensing receptor from bovine parathyroid. Nature $1993 \mathbf{3 6 6}$ 575-580.

11 Fan G, Goldsmith PK, Collins R, Dunn CK, Krapcho KJ, Rogers KV et al. N-linked glycosylation of the human $\mathrm{Ca}^{2+}$ receptor is essential for its expression at the cell surface. Endocrinology 1997138 1916-1922.

12 Bai M, Trivedi S, Kifor O, Quinn SJ \& Brown EM. Intermolecular interactions between dimeric calcium-sensing receptor monomers are important for its normal function. PNAS $1999 \mathbf{9 6}$ $2834-2839$.
13 Ward DT, Brown EM \& Harris HW. Disulfide bonds in the extracellular calcium-polyvalent cation-sensing receptor correlate with dimer formation and its response to divalent cations in vitro. Journal of Biological Chemistry 1998273 14476-14483.

14 Li Y, Song YH, Rais N, Connor E, Schatz D, Muir A et al. Autoantibodies to the extracellular domain of the calcium sensing receptor in patients with acquired hypoparathyroidism. Journal of Clinical Investigation 199697 910-914.

15 Goswami R, Srikanta SS \& Kochupillai N. Prevalence and significance of pancreatic islet cell and adrenal antibodies in patients with Graves' disease. Indian Journal of Medical Research $1995101201-206$.

16 Goswami R, Bhatia M, Goel R \& Kochupillai N. Reversible peripheral neuropathy in idiopathic hypoparathyroidism. Acta Neurologica Scandinavica $2002 \mathbf{1 0 5} 128-131$.

17 Goswami R, Gupta N, Goswami D, Marwaha R, Tandon N \& Kochupillai N. Prevalence and significance of low $25(\mathrm{OH}) \mathrm{D}$ in normal subjects in Delhi, North India. American Journal of Clinical Nutrition $2000 \mathbf{7 2} 472-475$.

18 Goswami R, Kochupillai N, Crock PA, Jaleel A \& Gupta N. Pituitary autoimmunity in patients with Sheehan's syndrome. Journal of Clinical Endocrinology and Metabolism $2002 \mathbf{8 7}$ $4137-4141$.

19 Kifor O, Moore FD Jr, Wang P, Goldstein M, Vassilev P, Kifor I et al. Reduced immunostaining for the extracellular $\mathrm{Ca}^{2+}$-sensing receptor in primary and uremic secondary hyperparathyroidism. Journal of Clinical Endocrinology and Metabolism $1996 \mathbf{8 1}$ 1598-1606.

20 McNeil L, Hobson S, Nipper V \& Rodland KD. Functional calciumsensing receptor expression in ovarian surface epithelial cells. American Journal of Obstetrics and Gynecology $1998 \mathbf{1 7 8}$ 305-313.

21 Canaff L, Petit JL, Kisiel M, Watson PH, Gascon-Barre M \& Hendy GN. Extracellular calcium-sensing receptor is expressed in rat hepatocytes. Coupling to intracellular calcium mobilization and stimulation of bile flow. Journal of Biological Chemistry 2001 276 4070-4079.

22 Wang R, Xu C, Zhao W, Zhang J, Cao K, Yang B et al. Calcium and polyamine regulated calcium-sensing receptors in cardiac tissues. European Journal of Biochemistry 2003270 2680-2688.

23 Rani R, Mukherjee R \& Stastny P. Diversity of HLA-DR2 in North Indians: the changed scenario after the discovery of DRB $1 * 1506$. Tissue Antigens 199852 147-152.

24 Rani R, Sood A, Lazaro AM \& Stastny P. Associations of MHC class II alleles with insulin-dependent diabetes mellitus (IDDM) in patients from North India. Human Immunology 199960 524-531.

25 Tiwari JL \& Teraski PI. HLA and Disease Association. New York: Springer \& Verlag, 1985.

26 Arlt W, Fremerey C, Callies F, Reincke M, Schneider P, Timmermann Wet al. Well-being, mood and calcium homeostasis in patients with hypoparathyroidism receiving standard treatment with calcium and vitamin D. European Journal of Endocrinology $2002146215-222$.

27 Illum F \& Dupont E. Prevalences of CT-detected calcification in the basal ganglia in idiopathic hypoparathyroidism and pseudohypoparathyroidism. Neuroradiology $1985 \mathbf{2 7} 32-37$.

28 Huddle KR \& Ally R. Idiopathic hypoparathyroidism in black South Africans. Quarterly Journal of Medicine 198970 53-60.

29 Forman MB, Sandler MP, Danziger A \& Kalk WJ. Basal ganglia calcification in postoperative hypoparathyroidism. Clinical Endocrinology $1980 \mathbf{1 2}$ 385-390.

30 Fattorossi A, Aurbach GD, Sakaguchi K, Cama A, Marx SJ, Streeten EA et al. Anti-endothelial cell antibodies: detection and characterization in sera from patients with autoimmune hypoparathyroidism. PNAS $1988 \mathbf{8 5}$ 4015-4019.

31 Uibo R, Aavik E, Peterson P, Perheentupa J, Aranko S, Pelkonen R et al. Autoantibodies to cytochrome P450 enzymes P450scc, $\mathrm{P} 450 \mathrm{c} 17$, and $\mathrm{P} 450 \mathrm{c} 21$ in autoimmune polyglandular disease 
types I and II and in isolated Addison's disease. Journal of Clinical Endocrinology and Metabolism 199478 323-328.

32 Tanaka H, Perez MS, Powell M, Sanders JF, Sawicka J, Chen S et al. Steroid 21-hydroxylase autoantibodies: measurements with a new immunoprecipitation assay. Journal of Clinical Endocrinology and Metabolism 199782 1440-1446.

33 Bjerneroth G, Juhlin C, Rastad J, Akerstrom G \& Klareskog L. MHC class I and II antigen expression on parathyroid cells and prospects for their allogenic transplantation. Transplantation $199356717-721$.

34 Bliddal H, Hegedus L, Hansen JM, Bech K, van der Gaag R \& Drexhage HA. The relationships between serum $\mathrm{T}_{3}$ index, thyroid volume, and thyroid stimulating, TSH receptor binding and thyroid growth stimulating antibodies in untreated Graves' disease. Clinical Endocrinology 198727 75-84.

35 Oosterhuis HJ, Limburg PC \& Hummel-Tappel E. The TH antiacetylcholine receptor antibodies in myasthenia gravis. Part 2. Clinical and serological follow-up of individual patients. Journal of Neurological Sciences $1983 \mathbf{5 8} 371-385$.

36 Kifor O, Moore FD Jr, Delaney M, Garber J, Hendy GN, Butters R et al. A syndrome of hypocalciuric hypercalcemia caused by autoantibodies directed at the calcium-sensing receptor. Journal of Clinical Endocrinology and Metabolism 200388 60-72.

37 Lovlie R, Eiken HG, Sorheim JI \& Boman H. The $\mathrm{Ca}^{(2+)}$-sensing receptor gene (PCAR1) mutation T151M in isolated autosomal dominant hypoparathyroidism. Human Genetics $1996 \mathbf{9 8}$ 129-133.

38 Sunthornthepvarakul T, Churesigaew S \& Ngowngarmratana S. A novel mutation of the signal peptide of the preproparathyroid hormone gene associated with autosomal recessive familial isolated hypoparathyroidism. Journal of Clinical Endocrinology and Metabolism $1999843792-3796$.

39 Goswami R, Kochupillai N, Gupta N, Kukreja A, Lan M \& Maclaren NK. Islet cell autoimmunity in youth onset diabetes mellitus in Northern India. Diabetes Research and Clinical Practice $20015347-54$.

Received 26 May 2003

Accepted 24 September 2003 